\title{
Retracted: The Role of Immune System Cells in Fracture Healing: Review of the Literature and Current Concepts
}

\author{
Dimitrios A. Flevas ${ }^{1}$, Maria G. Papageorgiou ${ }^{2}$, Panagiotis Drakopoulos ${ }^{1}$, George I. Lambrou ${ }^{1,} 3$ \\ 1. Laboratory for the Research of Musculoskeletal Disorders, National and Kapodistrian University of Athens, Athens, \\ GRC 2. Department of Pathology, University of Ioannina, Ioannina, GRC 3. First Department of Pediatrics, Choremeio \\ Research Laboratory, National and Kapodistrian University of Athens, Athens, GRC
}

Corresponding author: Dimitrios A. Flevas, dflevas@hotmail.com

Corresponding author: Dimitrios A. Flevas

\begin{abstract}
1. Laboratory for the Research of Musculoskeletal Disorders, National and Kapodistrian University of Athens, Athens, GRC 2. Department of Pathology, University of loannina, loannina, GRC 3. First Department of Pediatrics, Choremeio Research Laboratory, National and Kapodistrian University of Athens, Athens, GRC

How to cite this retraction

Flevas D A, Papageorgiou M G, Drakopoulos P, et al. (June 07, 2021) Retraction: The Role of Immune System Cells in Fracture Healing: Review of the Literature and Current Concepts. Cureus 13(6): r32. doi:10.7759/cureus.r32
\end{abstract}

\section{Retraction Notice}

This article has been retracted at the request of the authors. After careful consideration by our editorial staff, we have decided to grant the authors' request and retract the article. The formal request for retraction has been included below:

Dear Editorial,

This is to request the retraction of our article entitled entitled " The Role of Immune System Cells in Fracture Healing: Review of the Literature and Current Concepts” (ID: 20210426-13866-4cva89). As we have stressed in our previous communication on April the 30th we have found some deficits in its content and in particular, significant information not mentioned, as well as significant up-to-date knowledge omitted. Some up-todate information was not included in our manuscript, as well as we felt that it was not scientifically solid. As the topic was very specialized, significant parts could have been added, as well as several aspects were not presented in a good scientific way. In several parts of the manuscript, the language was not correctly stated and we felt that it was not presented in the highest possible level. In its current form, this articles does not represent the work we had in mind prior to publication.

We think that a revised work, would greatly improve the manuscript and therefore it is imperative to be able to expand it and improve its presentation. This revised work would update the current paper in order for the publication to be at the highest possible level. It is our belief that a new version of the paper will be for the benefit both for us and the scientific community.

For these reasons, we would like to request the retraction of the aforementioned publication, as we will attempt to rewrite it and present it in a more scientifically solid way.

I remain at your disposal for anything further.

Article published 04/26/2021 Retracted 06/07/2021
Best Regards,

\section{George I. Lambrou}

National and Kapodistrian University of Athens

First Department of Pediatrics

Choremeio Research Laboratory

Athens, Greece 